Rapid Reviews COVID-19

\title{
Reviews of "Quantitative Assessment of Olfactory Dysfunction Accurately Detects Asymptomatic COVID-19 Carriers"
}

Christopher von Bartheld ${ }^{1}$, Rafal Butowt, Danielle Reed ${ }^{2}$, Dolores Malaspina ${ }^{3}$

${ }^{1}$ University of Nevada Reno, Physiology and Cell Biology, ${ }^{2}$ Monell Chemical Senses Center, ${ }^{3}$ Professor, Icahn School of Medicine at Mount Sinai, Psychiatry, Neuroscience, Genetics Genomics

Published on: Sep 15, 2020

DOI: $10.1162 / 2 \mathrm{e} 3983 f 5.5 \mathrm{~d} 4560 \mathrm{cb}$

License: Creative Commons Attribution 4.0 International License (CC-BY 4.0). 
To read the original manuscript, click the link above.

Summary of Reviews: This study offers a quantitative approach for identifying asymptomatic COVID-19 patients paired with a diagnostic device optimized for use in the current pandemic. The claims are reliable, but minor follow up studies will better define implementation constraints.

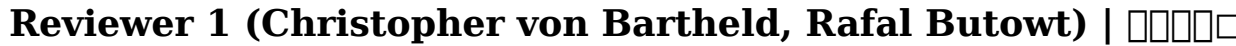

Reviewer 2 (Danielle Reed) |

\section{Reviewer 3 (Dolores Malaspina) |}

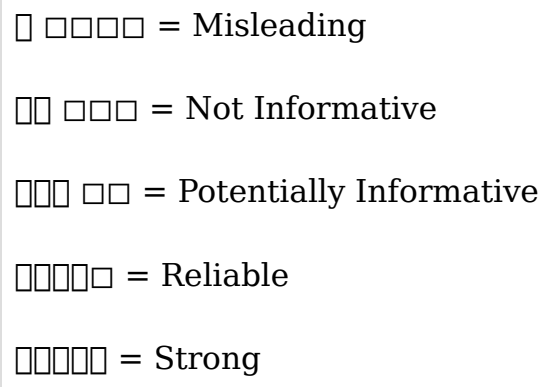

To read the reviews, click the links below. 